\title{
Quenching of metastable states of antiprotonic helium atoms by collisions with $\mathbf{H}_{2}$ molecules
}

\author{
B. Ketzer, F. J. Hartmann, T. von Egidy, C. Maierl, R. Pohl \\ Physik-Department, Technische Universität München, D-85747 Garching, Germany \\ J. Eades, E. Widmann*, T. Yamazaki ${ }^{\dagger}$ \\ CERN, CH-1211 Geneva 23, Switzerland \\ M. Kumakura, N. Morita \\ Institute for Molecular Science, Myodaiji, Okazaki 444, Japan \\ R. S. Hayano, M. Hori, T. Ishikawa, H. A. Torii \\ Department of Physics, University of Tokyo, 7-3-1 Hongo, Bunkyo-ku, Tokyo 113, Japan \\ I. Sugai \\ Institute for Nuclear Study, University of Tokyo, 3-2-1 Midori-cho, Tanashi, Tokyo 188, Japan \\ D. Horváth \\ Central Research Institute for Physics, Research Institute for Particle and Nuclear Physics, H-1525 Budapest, Hungary \\ (February 19, 1998)
}

\begin{abstract}
Laser resonance transitions between normally metastable states of antiprotonic helium atoms were induced making use of state dependent quenching effects caused by trace admixtures of $\mathrm{H}_{2}$ to the target helium gas. With this method of " $\mathrm{H}_{2}$-assisted inverse resonances" the decay rates of the states $(n, l)=(39, l), l=36,37,38$ and $(38, l), l=35,36,37$ of $\overline{\mathrm{pHe}}{ }^{+}$were determined as a function of the $\mathrm{H}_{2}$ admixture. The quenching cross sections at $30 \mathrm{~K}$ deduced therefrom for the states with $n=39$ were found to be of the order of the geometrical cross section for $\overline{\mathrm{p}} \mathrm{He}^{+}-\mathrm{H}_{2}$ collisions $\left(2 \cdot 10^{-15} \mathrm{~cm}^{2}\right)$, with a moderate decrease with increasing $l$. Within a given cascade with constant $v=n-l-1$, the quenching cross sections for states with $n=38$ are smaller by a factor of $4-6$ than those for states with $n=39$.
\end{abstract}

PACS number(s): 36.10.-k, 34.90.+q, 42.62.Fi

\section{INTRODUCTION}

About three per cent of antiprotons stopped in helium are known to survive for several microseconds ${ }^{1-6}-$ more than six orders of magnitude longer than the typical picosecond lifetimes of antiprotons brought to rest in ordinary matter. In all other target materials investigated so far ( $\mathrm{Li}, \mathrm{Ne}, \mathrm{Kr}, \mathrm{Xe}$ ) no evidence for delayed annihilation was seen ${ }^{3,4,6}$. This extraordinary longevity of antiprotons brought to rest in dense helium indicated the trapping of these antiprotons in certain metastable states of antiprotonic helium atoms.

The antiprotonic helium atom is a neutral three-body system $\overline{\mathrm{p}}-\mathrm{e}^{-}-\mathrm{He}^{2+}\left(\equiv \overline{\mathrm{pHe}}{ }^{+}\right)$formed when an antiproton $(\overline{\mathrm{p}})$, after its slowing down in inelastic collisions with atomic electrons, is captured by a helium atom, thereby liberating one of its two electrons. In the initially formed state of $\overline{\mathrm{p}} \mathrm{He}^{+}$the antiproton wavefunction is characterized by maximum overlap with the wavefunction of the electron it displaces, its most probable orbit having therefore a principal quantum number $n \sim n_{0} \equiv \sqrt{M / m} \simeq 38$, where $M$ and $m$ are the reduced masses of the $\overline{\mathrm{p}}-{ }^{4} \mathrm{He}^{2+}$ and $\mathrm{e}^{-}-{ }^{4} \mathrm{He}^{2+}$ system, respectively; the remaining electron stays essentially in the $1 \mathrm{~s}$ ground state. In this configuration the antiproton and the electron nearly equally share the total binding energy, $\sim 4 \mathrm{Ry}+I_{0}=+79 \mathrm{eV}$. On the above accounts, the $\overline{\mathrm{pHe}}{ }^{+}$system can be regarded as an exotic atom with two negatively charged particles bound by a helium nucleus, or as a peculiar diatomic one-electron molecule with the antiproton as a negative nucleus, the vibrational motion of the two nuclei, characterized by a vibrational quantum number $v=n-l-1$, being separable from the electronic motion. 
Since the wavefunction of the antiproton in its initially occupied state considerably overlaps that of the remaining electron, the antiproton was commonly believed to quickly eject the electron via Auger effect. The ionized $\overline{\mathrm{p}} \mathrm{He}^{2+}$ system thus formed is subject to strong electric fields in collisions with surrounding helium atoms, which induce Stark transitions to $n S$ states. These are immediately followed by annihilation of the antiproton with the nucleus, so that the whole process from capture to annihilation should be over in less than a picosecond.

Recent experiments ${ }^{7-12}$, which used laser light to specifically tag certain states of $\overline{\mathrm{p}} \mathrm{He}^{+}$, unambiguously proved that the metastability observed in helium occurs due to antiprotons being captured in high- $n$ states with large angular momentum $l \lesssim n-1$. For these circular or nearly circular orbits, fast Auger transitions with multipolarity $L=\Delta l<3$ are energetically impossible. This is due to the small energy level spacing $\Delta E=E_{n}-E_{n-1}$ of $\sim 2 \mathrm{eV}$ for $n=38$, which requires a large jump in the principal quantum number $n$ of the system in order to release the energy necessary for ejection of the second electron $\left(I_{0} \sim 79 \mathrm{eV}-4 \mathrm{Ry}=24.6 \mathrm{eV}\right)$. Since $l \lesssim n-1$ for circular or nearly circular orbits, this is equivalent to a large jump in orbital angular momentum $l$. Auger transitions with $\Delta l>3$, on the other hand, are strongly suppressed, because the Auger rate, which is known to decrease by three orders of magnitude when $|\Delta l|$ increases by 1 , is much lower than the radiative rate ${ }^{13-16}$. The continued presence of this electron suppresses collisional Stark mixing of high- $l$ and low-l states by removing the $l$-degeneracy of states with equal $n$ and, due to the Pauli exclusion principle, preventing the electron cloud of a colliding helium atom from penetrating the $\overline{\mathrm{p}} \mathrm{He}^{+}$system. Hence, these high- $(n, l)$ states predominantly decay via slow radiative dipole transitions with $\Delta l= \pm 1$, whose rate as given by Fermi's Golden Rule is

$$
\lambda=\frac{4}{3} \frac{\left(\Delta E_{n}\right)^{3}}{\hbar^{4} c^{3}}|\langle f|E 1| i\rangle|^{2} .
$$

Due to the almost classical localization of the antiproton wavefunction for large- $n$ states, transitions with $\Delta n>1$ are much slower than those with $\Delta n=1$, because the larger transition energies $\Delta E_{n}$ for $\Delta n>1$ are outweighed by a much smaller overlap integral $\langle f|E 1| i\rangle$ of initial and final state wavefunctions ${ }^{13}$. In the molecular picture, this means that the familiar selection rule $\Delta v=\Delta(n-l-1)=0,1$ for radiative transitions between low-lying vibrational states of an ordinary diatomic molecule is reduced to $\Delta v=0$ in the case of $\overline{\mathrm{pHe}}{ }^{+}$ due to the large angular momentum ${ }^{14}$. Thus, as our laser spectroscopy experiments in pure helium confirm, the metastable states in the large- $(n, l)$ region preferably decay via a cascade of slow radiative transitions with constant vibrational quantum number $v$, each with a lifetime of $\sim 1 \mu \mathrm{s}$. The cascade terminates when the antiproton arrives at a short-lived state from which Auger decay with $\Delta l \leq 3$ is energetically possible. In the calculated level scheme of large- $(n, l)$ states of $\overline{\mathrm{p}}^{4} \mathrm{He}^{+}$, displayed in Fig. 1, these sequences of transitions are shown as arrows along $v=$ const with the theoretical transition wavelengths given in units of $\mathrm{nm}$. The full horizontal bars denote radiation-dominated metastable states while the zigzag bars indicate Auger-dominated short-lived states, which decay to the states of ionized $\overline{\mathrm{p}} \mathrm{He}^{2+}$, shown as dash-dotted lines.

The anomalously long antiproton lifetime thus produced in pure helium has been observed to be reduced slightly by $10 \%$ admixtures of light noble gases (Ne, Ar, $\mathrm{Kr}$ ), whereas already $250 \mathrm{ppm}$ of Xe showed a much stronger influence ${ }^{6}$. For the molecular admixtures $\mathrm{H}_{2}$ and $\mathrm{O}_{2}$, however, the quenching was found to be much more violent: Already a few hundred ppm destroyed the metastability almost completely. On the other hand, the presence of $\sim 1 \%$ of $\mathrm{N}_{2}$ affected the $\overline{\mathrm{p}}$ lifetime much less than $\mathrm{O}_{2}$ and $\mathrm{H}_{2}{ }^{2,3,6}$. It is the purpose of the present investigation to shed some new light on the physics of these different quenching processes. In the case of $\mathrm{H}_{2}$, even at concentrations of a few ppm a fast-decaying component appeared in the delayed annihilation time spectrum (DATS) together with a corresponding reduction of the long-lived part, indicating that certain normally metastable states had been converted to shorter-lived ones. A more detailed investigation of the effect of $\mathrm{H}_{2}$ on populations and lifetimes of individual cascades or single states of $\overline{\mathrm{p}} \mathrm{He}^{+}$, rather than on the average lifetime of antiprotons as reflected by the DATS, required the application of a laser resonance technique, which we had especially developed for studying these antiprotonic helium atoms. Its principle is to induce resonance transitions of $\overline{\mathrm{pHe}}^{+}$from a metastable state to a short-lived one by irradiation with a laser pulse of the corresponding wavelength, resulting in almost instantaneous annihilation of the antiproton and a spike-like response in the DATS at the time the laser is fired ${ }^{15}$. In addition to yielding very accurate values for the transition wavelengths, this method also provided the means to determine the lifetimes of metastable levels and investigate their pressure and impurity dependence ${ }^{19-21}$.

Studying the lifetimes of the states $(n, l)=(37,34)$ at the end of the cascade $v=2$ and $(n, l)=(39,35)$ at the end of $v=3$ as a function of the $\mathrm{H}_{2}$-concentration, we had observed a strong $n$ dependence of the quenching effect ${ }^{20}$ indicating that in these cascades states with higher $n$ are much more strongly quenched by collisions with $\mathrm{H}_{2}$ molecules than those with lower $n$. Recently, we employed these state-dependent quenching effects to selectively depopulate the higher one of two adjacent metastable levels in a cascade by adding the appropriate concentration of $\mathrm{H}_{2}$ molecules to the target gas and then applied a laser pulse to induce an "inverse" transition $(n, l) \rightarrow(n+1, l+1)$ between the long-lived lower level and the now short-lived upper one ${ }^{22}$. This " $\mathrm{H}_{2^{-}}$ assisted inverse resonance" (HAIR) method extended our laser resonance technique (restricted in the pure helium case to transitions between a metastable and a short- 
lived level at the end of a cascade) to previously inaccessible levels in the metastable region of the $(n, l)$-plane. The partial level scheme of $\overline{\mathrm{p}}^{4} \mathrm{He}^{+}$, shown in the inset of Fig. 1, summarizes the six HAIR transitions (bold arrows $(n, l)=(38, l) \rightarrow(39, l+1), l=35,36,37$ and $(n, l)=(37, l) \rightarrow(38, l+1), l=34,35,36$ between normally metastable states (bold horizontal bars) and the two "conventional" metastable to short-lived transitions (thin arrows) at the end of the cascades $v=2$ and 3 , together with the experimental transition wavelengths in units of $\mathrm{nm}$. The $(n, l)$ assignments of the two "conventional" transitions were made taking into acount a theoretical estimate by Ohtsuki ${ }^{15}$ of the boundary between the radiation-dominated metastable states and the Auger-dominated short-lived ones, while the HAIR transitions were assigned their respective $(n, l)$ values by comparing the experimental transition wavelength with the theoretical results by Korobov ${ }^{17,18}$.

In order to identify the HAIR transitions as peaks in the DATS, it was necessary to find, for each individual transition, the appropriate $\mathrm{H}_{2}$ concentration which, on the one hand, considerably shortened the lifetime of the upper level, but, on the other, still left a sufficiently large population in the lower one. Employing these $\mathrm{H}_{2}$ concentration scans we were able to determine the lifetimes and $\mathrm{H}_{2}$ quenching cross sections of the states $(38, l), l=35,36,37$ and $(39, l), l=36,37,38$ presented here for the first time, which were previously known only for the two states $(37,34)$ and $(39,35)$, thus providing important systematic information on the $l$ and $n$ dependence of the $\mathrm{H}_{2}$ quenching for a wider variety of metastable states of $\overline{\mathrm{p}} \mathrm{He}^{+}$.

In the present paper we give a brief description of the experimental setup, describe the procedure used to determine level decay rates from the HAIR measurements and present $\mathrm{H}_{2}$ quenching cross sections for all the six daughter states of the observed HAIR transitions deduced therefrom.

\section{EXPERIMENT}

The results reported here were obtained with the $200 \mathrm{MeV} / \mathrm{c}$ antiproton beam from CERN's Low Energy Antiproton Ring LEAR. The HAIR method requires that the laser pulse be applied early enough after the antiproton stop to assure that there is still a detectable population left in the lower level, since the lower level lifetime is shortened to some extent by the $\mathrm{H}_{2}$ admixture as well as that of the upper one. This condition was met by using a sequence of $100 \mathrm{~ns}$ long antiproton bunches, each containing $\sim 10^{8}$ particles. The signal that initiated the extraction of antiprotons was used to trigger our laser system prior to the arrival of the bunch. Thus the antiprotonic helium atoms could be probed as early as $180 \mathrm{~ns}$ after their formation, in contrast to the case of continuous extraction of antiprotons, where the laser system was triggered by a randomly arriving antiproton and hence could be fired only after an intrinsic ignition time of $1.3 \mu \mathrm{s}$.

Figure 2 shows a plan view of the experimental setup used for the HAIR measurements. At the end of the beam line the bunch of antiprotons passed through a $100 \mu \mathrm{m}$ thick Be window and traversed a few $\mathrm{cm}$ of air and a parallel plate ionization chamber (PPIC), which served as a beam position and intensity monitor, before entering the target chamber through a $50 \mu \mathrm{m}$ Kapton and a $500 \mu \mathrm{m}$ CuBe window. To stop the antiprotons in the target, $1150 \mu \mathrm{m}$ of degrader material (EUPLEX) were added between the Kapton and the CuBe window for moderation. Additional $175 \mu \mathrm{m}$ of EUPLEX were placed outside the cryostat in front of the CuBe window for the fine adjustment of the antiproton stopping distribution inside the target gas chamber. The total thickness of degrader material necessary was determined by a Monte Carlo simulation taking into account the Barkas effect on the energy $\operatorname{loss}^{3}$. The pulsed dye laser beam entered the target chamber through two quartz windows on the side opposite to the beam entrance window.

The target gas consisted of ${ }^{4} \mathrm{He}(99.9996 \%$ pure $)$ to which $\mathrm{H}_{2}$ (99.999\% pure) had been premixed at concentrations of $98.6 \mathrm{ppm}, 291 \mathrm{ppm}$ and $968 \mathrm{ppm}$ with a relative accuracy of $2 \%$. To avoid $\mathrm{H}_{2}$ condensation in the target gas and contamination by impurities such as $\mathrm{N}_{2}$ or $\mathrm{O}_{2}$, the target gas was maintained at a temperature of $30 \mathrm{~K}$ throughout the experiment. For this purpose the stainless steel target chamber was mounted inside a cryostat with cold helium flowing through Cu pipes surrounding the target vessel. To stabilize the temperature a heating wire around the target chamber was connected in a feedback loop to a sensor located on the target chamber. The number density of $\mathrm{H}_{2}$ molecules in the target gas could be changed by varying the pressure of the premixed gases between 1.5 bar and 10 bar. The pressure dependence of the metastability in the low density regime employed here is known to be negligible ${ }^{19}$ compared with the violent dependence on the $\mathrm{H}_{2}$ concentration.

Each antiproton bunch of some $10^{8}$ particles produces

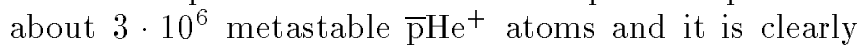
impossible to measure individual annihilations of these within $10 \mu \mathrm{s}$. We therefore used an "analog" method to record the DATS ${ }^{12,23}$ : the Cerenkov light produced by the charged annihilation products in a simple Lucite radiator, subtending a solid angle of about $36 \%$ of $4 \pi$, was detected by a highly linear, fast photomultiplier tube (PMT) and the resulting anode current was recorded by a $400 \mathrm{MHz}$ digital oscilloscope (2GS/s, 8 bit resolution). Since the large light pulse coming from $\sim 10^{8}$ antiprotons annihilating within the first 10 ns would damage a normal PMT, we used a gatable variety, developed by HAMAMATSU Photonics Ltd. (R5504GX) for this specific application. 


\section{DECAY RATES}

Figure 3(a) shows an analog DATS obtained at $30.2 \mathrm{~K}$, 4.8 bar and an $\mathrm{H}_{2}$ concentration of $98.6 \mathrm{ppm}$ with the laser wavelength tuned to the $(n, l)=(38,35) \rightarrow(39,36)$ transition. A small but significant peak appeared in the DATS at exactly the laser firing time $(\sim 180 \mathrm{~ns}$ after arrival of the antiproton bunch). The time structure of the corresponding laser pulse recorded by a PIN diode can be seen in Fig. 3(b). For the duration of the laser pulse $(\sim 30 \mathrm{~ns})$, the resonance peak in the DATS not only reflects the daughter state lifetime, but is distorted by Rabi oscillations and dephasing collisions ${ }^{15}$. In addition, it is convoluted with the complicated time structure of the laser pulse itself. After the end of the laser pulse, however, the peak decays with a rate determined only by the decay rate of the short-lived upper level of the HAIR transition. In our analysis of the peak decay rates we therefore confined ourselves to times after the end of the laser pulse.

In pulsed extraction, the analog DATS observed without laser irradiation reflects the chain decay of many independent antiproton cascades from all initially populated levels, superimposed on a single exponential decay component with a lifetime of $2.2 \mu \mathrm{s}$, originating from the $\pi^{+} \rightarrow \mu^{+} \rightarrow \mathrm{e}^{+}$decay chain of prompt annihilation pions stopped in material surrounding the target chamber. The analog detection of Čerenkov light produced in a large radiator does not permit these $\mu^{+}$decays to be be distinguished from delayed annihilation pions with our present experimental setup.

In order to reliably determine the decay rates of the laser-induced peaks, an adequate description of this "background" (here, "background" means analog DATS without laser irradiation) by a simplified model as compared to the multi-parameter analytical solution of the cascade equations is essential. It turned out that a simple sum of two exponentials can reproduce the background very well:

$$
B(t)=N\left[\lambda_{1} f e^{-\lambda_{1} t}+\lambda_{2}(1-f) e^{-\lambda_{2} t}\right],
$$

where $\lambda_{1}$ was fixed to $(2.2 \mu \mathrm{s})^{-1}$, the decay rate of stopped $\mu^{+}$, while $\lambda_{2}$ was a free parameter of the fit; $f$ denotes the fraction of the $2.2 \mu$ s contribution to the total spectrum and $N$ is an overall normalization parameter.

For times $t>t_{\mathrm{L}}^{\text {off }}$ a single exponential with an amplitude $A_{\mathrm{p}}$ and a decay rate $\lambda_{\mathrm{p}}$ was added to this background to describe the exponentially decaying laserinduced peak:

$$
\begin{aligned}
& A(t)= \\
& \begin{cases}N\left[\lambda_{1} f e^{-\lambda_{1} t}+\lambda_{2}(1-f) e^{-\lambda_{2} t}\right], & t<t_{\mathrm{L}}^{\text {on }} \\
N\left[\lambda_{1} f e^{-\lambda_{1} t}+\lambda_{2}(1-f) e^{-\lambda_{2} t}+A_{\mathrm{p}} \lambda_{\mathrm{p}} e^{-\lambda_{\mathrm{p}} t}\right], & t>t_{\mathrm{L}}^{\mathrm{off}}\end{cases}
\end{aligned}
$$

Here, $t_{\mathrm{L}}^{\text {on }}$ and $t_{\mathrm{L}}^{\text {off }}$ denote the beginning and the end of the laser pulse, respectively.

Even this relatively simple function, however, yielded consistent fit results only for high-statistics analog DATS which had been recorded at the beginning of a spill from LEAR, whereas fairly largely fluctuating results were obtained for medium- and low-statistics spectra with less antiprotons in a bunch. In order to include all existing analog DATS for a given transition recorded with the same target conditions in our analysis and to eliminate fluctuations in the fit result, a set of analog DATS was fitted simultaneously with the same parameter values for $\lambda_{1}, \lambda_{\mathrm{p}}$ and $f$, respectively, for each spectrum. Only the peak amplitudes $A_{\mathrm{p}}^{i}$ and the normalizations $N^{i}$ were treated as independent parameters for each spectrum $i$. Hence, depending on the number $m$ of DATS included in the fit, the number of free parameters was $3+2 m$. The fit was performed in several steps:

1. Fitting of the background function Eq. (2) to the region before and after the peak to obtain initial values for the parameters $\lambda_{2}, f$ and $N^{i}$.

2. Fitting of Eq. (3) to the entire region, excluding the $30 \mathrm{~ns}$ duration of the laser pulse, with the parameters $\lambda_{2}, f$ and $N^{i}$ fixed to the values obtained in step 1 to get preliminary values for the peak parameters $\lambda_{\mathrm{p}}$ and $A_{\mathrm{p}}^{i}$.

3. Release of all parameters and fitting of Eq. (3) with the complete set of parameters to the spectrum, again excluding the $30 \mathrm{~ns}$, during which the laser pulse was applied.

Figure 3(a) shows an analog DATS with the result of a simultaneous fit of 27 analog DATS taken at $30.2 \mathrm{~K}$, 4.80 bar and an $\mathrm{H}_{2}$ concentration of $98.6 \mathrm{ppm}$ with the laser tuned to the transition $(n, l)=(38,35) \rightarrow(39,36)$ (solid line). The vertical lines indicate the duration of the laser irradiation, which has been excluded from the fit. The background function without the peak is also shown (dashed line).

In Fig. 4 background-subtracted peaks of the transition $(n, l)=(38,35) \rightarrow(39,36)$ at three different number densities of $\mathrm{H}_{2}$ molecules are displayed. The lifetime $\tau$, with which the peak decays after the end of the laser pulse, clearly becomes shorter with increasing $\mathrm{H}_{2}$ admixture, directly reflecting the reduced daughter state lifetime of the transition. Table I summarizes the decay rates of the states $(39, l), l=36,37,38$ and $(38, l), l=35,36,37$, deduced at different target conditions. The number density of $\mathrm{H}_{2}$ molecules $n_{\mathrm{H}_{2}}$ was calculated using the second order virial expansion of the state equation. The second virial coefficients were interpolated from theoretical values $^{24}$.

In order to obtain correct errors for the fitted decay rates it was essential to assign uncertainties to the sampled analog DATS. Generally, the statistical fluctuations observed in a measurement of a signal $V=N \cdot \bar{v}$ generated by $N$ events, each producing an average pulse height 
$\bar{v}$, arise from two sources: (i) the statistical fluctuations $\sigma_{N}=\sqrt{N}$ of the number of events $N$, and (ii) the pulse height fluctuations $\sigma_{v}$ of a single event, in the case of a PMT readout due to the approximately statistical nature of the conversion of photons to photoelectrons. Therefore, $\sigma_{v}=\bar{v} / \sqrt{\bar{n}_{\mathrm{pe}}}$ with $\bar{n}_{\text {pe }}$ being the average number of photoelectrons produced by a single event. The variance of the observed signal $V$ is then ${ }^{25}$

$$
\sigma_{V}^{2}=N \sigma_{v}^{2}+\bar{v}^{2} \sigma_{N}^{2}=N \bar{v}^{2}\left(1+\frac{1}{\bar{n}_{\mathrm{pe}}}\right) .
$$

Since an exact calculation of the bin errors using Eq. (4) fails due to the lack of reliable data on the number of charged particles hitting the Čerenkov counter, the number of Cerenkov photons produced and the light collection efficiency in the radiator and the light guide, an attempt was made to determine the bin errors from an analysis of the statistical fluctuations in the measured analog DATS. To this end the reduced chi squared, $\chi_{\nu}^{2}$, was evaluated for each fit of Eq. (3) to a set of analog DATS using bin errors given by $\sigma_{i}=\sqrt{V_{i}}$ for each bin $i$. To account for the time response of the PMT system, which is governed by an electron drift time spread of the order of $5 \mathrm{~ns}$, the bin width was chosen to be $5 \mathrm{~ns}$ as well. In the short time range under consideration, Eq. (3) can be assumed to perfectly describe the analog DATS, as can be seen from Fig. 3(a). Assuming that the signal heights $V_{i}$ are independent from each other, the correct errors on the signal height are then $\hat{\sigma}_{i}=\sqrt{\chi_{\nu}^{2}} \cdot \sigma_{i}$, which yield a $\hat{\chi}_{\nu}^{2}$ of 1 . Application of this procedure to the spectrum shown in Fig. 3(a) resulted in a relative error on the signal height of $\hat{\sigma}_{i} / V_{i}=1.1 / \sqrt{V_{i}}$, where $V_{i}$ is measured in units of $\mathrm{mV}$.

In order to check this result, an estimate of the error based on Eq. (4) can be obtained, making the following assumptions: The average number of Čerenkov photons produced by a charged annihilation pion in $3 \mathrm{~cm}$ of Lucite can be calculated ${ }^{26}$ to be 1500 , taking into account the spectral sensitivity of the PMT and the well-known average energy distribution of annihilation pions. With an estimated light collection efficiency in the radiator and the light guide of $5 \%$ and a quantum efficiency of the photo cathode of 0.25 , the average number of photoelectrons produced by a single pion is $\bar{n}_{\text {pe }}=19$. Taking into account the known gain of the secondary electron multiplier section of the PMT, $g=10^{4}$, the average charge deposited on the anode of the PMT by an annihilation pion is $30 \cdot 10^{-15} \mathrm{C}$. Using Eq. (4), the relative error of the voltage at $50 \Omega$ in a time bin of $5 \mathrm{~ns}$ is then $\sigma_{V} / V=0.6 / \sqrt{V}$, with $V$ given in units of $\mathrm{mV}$. Considering the crude assumptions made, this estimate is in fair agreement with the results from the fluctuation analysis.

\section{QUENCHING CROSS SECTIONS}

Figure 5 presents the observed decay rates as a function of the number density of $\mathrm{H}_{2}$ molecules, exhibiting a fairly linear increase with increasing $\mathrm{H}_{2}$ number density. It is then justified to apply a simple collisional model, in which the metastable states are quenched in binary collisions with $\mathrm{H}_{2}$ molecules, the sensitivity to $\mathrm{H}_{2}$ being characterized by a state dependent quenching cross section $\sigma_{q}(n, l)$. Assuming a hard-sphere potential, the $\overline{\mathrm{pHe}}^{+}$system is slowed down from a kinetic energy of a few $\mathrm{eV}$ after its formation to thermal energies in about ten elastic collisions with other helium atoms ${ }^{27,28}$ and has therefore been thermalized before it encounters an $\mathrm{H}_{2}$ molecule. The observed decay rate of a given state $(n, l)$ can then be expressed $\mathrm{as}^{20}$

$$
\lambda(n, l)=\lambda_{0}(n, l)+n_{\mathrm{H}_{2}} v_{\mathrm{th}} \sigma_{\mathrm{q}}(n, l),
$$

where $n_{\mathrm{H}_{2}}$ is the number density of $\mathrm{H}_{2}$ molecules in the target gas and $v_{\mathrm{th}}=\sqrt{8 k_{\mathrm{B}} T / \pi M_{\mathrm{red}}}$ is the relative thermal velocity of the collision partners ${ }^{29}$. Here, $k_{\mathrm{B}}$ denotes the Boltzmann constant, $T$ the target gas temperature and $M_{\text {red }}$ the reduced mass of the $\bar{p} \mathrm{He}^{+}-\mathrm{H}_{2}$ system. Since the daughter states of the HAIR transitions are not accessible by our laser resonance method without the admixture of $\mathrm{H}_{2}$, experimental values for their decay rates in pure helium are not available. Therefore, the values used for $\lambda_{0}(n, l)$ were the sum of i) all radiative E1 transition rates between states $(n, l)$ and $(n+\Delta n, l \pm 1)$, obtained in a Born-Oppenheimer calculation ${ }^{14}$, and ii) the rate of Auger transitions from a level $(n, l)$ to a state of ionized $\overline{\mathrm{pHe}} \mathrm{H}^{2+}$, calculated with a variational method based on a molecular expansion of the wavefunction ${ }^{16}$. All calculations of decay rates available up to now, however, do not take into account the $\overline{\mathrm{p}} \mathrm{He}^{+}-\mathrm{He}$ interaction at finite target densities, which is known experimentally to shorten the lifetime of the state $(37,34)$ considerably ${ }^{19}$. For the state $(38,35)$, the lifetime shortening at our experimental conditions ( $30 \mathrm{~K}, 1.55$ bar) can be assumed to be $\lesssim 15 \%$ compared to the theoretical result, while the $(39,35)$ state has been observed to remain nearly unaffected up to liquid helium densities ${ }^{19}$. Hence, a $15 \%$ error was ascribed to the theoretical decay rates of the states under investigation ( $n=38,39, l \geq 35$ ) as an upper limit of a possible reduction of the level lifetimes at finite densities. In Fig. 5 the results of fitting Eq. (5) to the theoretical and experimental decay rates are shown as straight lines. In Table II we present the quenching cross sections of the different states deduced from this fit. To complete the picture, the quenching cross sections deduced earlier for the levels $(n, l)=(39,35)$ and $(37,34)$ at the end of the cascade $v=3$ and $v=2$, respectively, are also shown 20,21 .

The results give a quantitative confirmation of the strong $n$-dependence of the quenching by $\mathrm{H}_{2}$ molecules: within a given cascade, the lifetime of the upper state is more sensitive to $\mathrm{H}_{2}$ by a factor of $4-6$ than that of the 
lower state. It was this fact that facilitated the HAIR method. In addition, the quenching cross sections depend on the angular momentum quantum number $l$ : for constant principal quantum number $n$, states with higher $l$ are less affected by $\mathrm{H}_{2}$ admixtures than those with lower $l$. Figure 6 summarizes the observed $(n, l)$ dependence of the $\mathrm{H}_{2}$ quenching cross section $\sigma_{\mathrm{q}}$.

\section{DISCUSSION AND CONCLUSION}

The HAIR method has provided access to a class of metastable states in the $(n, l)$-plane that had previously been hidden from experiment. The decay rates of six transitions between such normally metastable states of $\overline{\mathrm{pHe}}^{+}$observed by this method were determined and quenching cross sections for the $\mathrm{H}_{2}$-induced short-lived daughter states were deduced. We observed a strong increase of the quenching cross sections with increasing principal quantum number $n$. On the other hand, the cross sections decrease moderately with increasing angular momentum quantum number $l$. Using the van der Waals radii of a $\mathrm{He}$ atom, $r_{\mathrm{He}}=1.22 \AA^{30}$, and an $\mathrm{H}_{2}$ molecule, $r_{\mathrm{H}_{2}}=1.38 \AA^{31}$, the geometrical cross section for a $\overline{\mathrm{pHe}}{ }^{+}-\mathrm{H}_{2}$ collision is $21 \cdot 10^{-16} \mathrm{~cm}^{2}$. The fact that the quenching cross sections deduced for states with $n=39$ are of the same order of magnitude as the geometrical cross section means that these states are quenched to almost $100 \%$ in a single collision with an $\mathrm{H}_{2}$ molecule, while the probability to quench the energetically lower lying states with $n=38$ is much smaller. Recent experiments with $\mathrm{O}_{2}$ admixtures exhibit an even more violent quenching of states than in the case of $\mathrm{H}_{2}$, however, with similar quenching cross sections of the order of the geometrical cross section for all metastable states $^{32}$. At present the physico-chemical interactions leading to the strong quenching of all levels in the case of $\mathrm{O}_{2}$ as well as the surprising state dependence of the quenching observed for $\mathrm{H}_{2}$ are not yet understood.

Here, we confine ourselves to qualitative arguments, aiming at a tentative explanation of the state dependent quenching in the case of $\mathrm{H}_{2}$ admixtures, which need further theoretical confirmation. The radial probability densities (charge distributions) of the antiproton and the electron in each of the six HAIR daughter states of $\overline{\mathrm{pHe}}^{+}$calculated by Korobov ${ }^{17,18,33}$, are shown as functions of the $\overline{\mathrm{p}}-\mathrm{He}^{2+}$ and $\mathrm{e}^{-}-\mathrm{He}^{2+}$ distances, respectively, in Fig. 7. These average charge distributions have been determined by integrating over all angular variables, thus neglecting the polarization of the electron cloud due to the Coulomb repulsion between the slowly moving antiproton and the electron ${ }^{13}$. Clearly, the mean radius of the antiproton orbit increases with increasing $n\left(\propto n^{2}\right.$ in the case of hydrogenic wave functions), while the mean electron radius decreases slightly. For states with constant $n$, the mean antiproton radius decreases with increasing $l$; the electron wavefunction is not altered con- siderably. The fact that the mean radius of the antiproton exhibits the same $(n, l)$ dependence as the observed quenching cross section, may indicate that the primary role in the quenching process is played by the antiproton. The electron, whose spatial distribution varies reciprocally with $(n, l)$ compared to that of the antiproton, shields the $\overline{\mathrm{p}}$ orbit of lower- $n$ states against a colliding $\mathrm{H}_{2}$ molecule more than that of higher- $n$ states, possibly leading to a reduced interaction probability of antiprotons in lower- $n$ states with a colliding $\mathrm{H}_{2}$ molecule. It remains an open question, how the exchange interaction between the remaining electron of the $\overline{\mathrm{p}} \mathrm{He}^{+}$system and the two electrons of the $\mathrm{H}_{2}$ molecule protects the metastable states of lower $n$, similar to the effect that leads to the Paulirepulsion between $\overline{\mathrm{pHe}}^{+}$and He mentioned in Sec. I., and whether such an effect can account for the state dependent quenching cross sections at all, which are a factor of 4-6 larger for states with $n=39$ than for those with $n=38$. Calculations of state dependent $\left[\overline{\mathrm{pHe}}{ }^{+}\right]_{n, l}-\mathrm{H}_{2}$ interaction potentials are therefore eagerly awaited.

\section{ACKNOWLEDGMENTS}

We are indebted to the LEAR and PS staff at CERN for their tireless dedication to providing us with the highquality antiproton beam, to Dr. V.I. Korobov for providing us with his theoretical results and to Hamamatsu Photonics Ltd. for developing the special gated PMT used in this experiment. The present work is supported by the German Bundesministerium für Bildung, Wissenschaft, Forschung und Technologie, the Grants-in-Aid for Specially Promoted Research and for International Scientific Research of the Japanese Ministry of Education, Science and Culture and the Hungarian National Science Foundation. H. A. T. acknowledges the support of the Japan Society for the Promotion of Science.

${ }^{1}$ M. Iwasaki, S.N. Nakamura, K. Shigaki, Y. Shimizu, H. Tamura, T. Ishikawa, R.S. Hayano, E. Takada, E. Widmann, H. Outa, M. Aoki, P. Kitching and T. Yamazaki, Phys. Rev. Lett. 67, 1246 (1991).

${ }^{2}$ T. Yamazaki, E. Widmann, R.S. Hayano, M. Iwasaki, S.N. Nakamura, K. Shigaki, F.J. Hartmann, H. Daniel, T. von Egidy, P. Hofmann, Y.-S. Kim and J. Eades, Nature 361, 238 (1993).

${ }^{3}$ S.N. Nakamura, R.S. Hayano, M. Iwasaki, K. Shigaki, E. Widmann, T. Yamazaki, H. Daniel, T. von Egidy, F.J. Hartmann, P. Hofmann, Y.-S. Kim and J. Eades, Phys. Rev. A49, 4457 (1994).

${ }^{4}$ E. Widmann, I. Sugai, T. Yamazaki, R. S. Hayano, M. Iwasaki, S.N. Nakamura, H. Tamura, T.M. Ito, A. Kawachi, N. Nishida, W. Higemoto, Y. Ito, N. Morita, 
F.J. Hartmann, H. Daniel, T. von Egidy, W. Schmid, J. Hoffmann and J. Eades, Phys. Rev. A51, 2870 (1995).

${ }^{5}$ B. Ketzer, F.J. Hartmann, H. Daniel, T. von Egidy, A. Niestroj, S. Schmid, W. Schmid, T. Yamazaki, I. Sugai, K. Nakayoshi, R.S. Hayano, F.E. Mass, H.A. Torii, T. Ishikawa, H. Tamura, N. Morita, D. Horváth, J. Eades and E. Widmann, Phys. Rev. A53, 2108 (1996).

${ }^{6}$ E. Widmann, I. Sugai, T. Yamazaki, R.S. Hayano, M. Iwasaki, S.N. Nakamura, H. Tamura, T.M. Ito, A. Kawachi, N. Nishida, W. Higemoto, Y. Ito, N. Morita, F.J. Hartmann, H. Daniel, T. von Egidy, W. Schmid, J. Hoffmann and J. Eades, Phys. Rev. A53, 3129 (1996).

${ }^{7}$ N. Morita, M. Kumakura, T. Yamazaki, E. Widmann, H. Masuda, I. Sugai, R.S. Hayano, F.E. Maas, H.A. Torii, F.J. Hartmann, H. Daniel, T. von Egidy, B. Ketzer, W. Müller, W. Schmid, D. Horvath and J. Eades, Phys. Rev. Lett. 72, 1180 (1994).

${ }^{8}$ R.S. Hayano, F.E. Maas, H.A. Torii, N. Morita, M. Kumakura, T. Yamazaki, H. Masuda, I. Sugai, F.J. Hartmann, H. Daniel, T. von Egidy, B. Ketzer, W. Müller, W. Schmid, D. Horvath, J. Eades and E. Widmann, Phys. Rev. Lett. 73, 1485 (1994); errata Phys. Rev. Lett. 73, 3181 (1994)

${ }^{9}$ F.E. Maas, R.S. Hayano, T. Ishikawa, H. Tamura, A. Torii, N. Morita, T. Yamazaki, I. Sugai, K. Nakayoshi, F.J. Hartmann, H. Daniel, T. von Egidy, B. Ketzer, A. Niestroj, S. Schmid, W. Schmid, D. Horváth, J. Eades and E. Widmann, Phys. Rev. A52, 4266 (1995).

${ }^{10}$ H.A. Torii, M. Hori, T. Ishikawa, F.E. Mass, R.S. Hayano, N. Morita, M. Kumakura, I. Sugai, B. Ketzer, H. Daniel, F.J. Hartmann, R. Pohl, R. Schmidt, T. von Egidy, D. Horváth, J. Eades, E. Widmann and T. Yamazaki, Phys. Rev. A53, R1931 (1996).

${ }^{11}$ R.S. Hayano, T. Ishikawa, H. Tamura, H.A. Torii, M. Hori, F.E. Mass, N. Morita, M. Kumakura, I. Sugai, F.J. Hartmann, H. Daniel, T. von Egidy, B. Ketzer, R. Pohl, D. Horváth, J. Eades, E. Widmann and T. Yamazaki, Phys. Rev. A55, R1 (1997).

${ }^{12}$ T. Yamazaki, E. Widmann, J. Eades, M. Kumakura, N. Morita, H.A. Torii, M. Hori, T. Ishikawa, F.E. Mass, H. Tamura, R.S. Hayano, I. Sugai, Y. Fujita, B. Ketzer, H. Daniel, F.J. Hartmann, M. Hasinoff, R. Pohl, R. Schmidt, T. von Egidy and D. Horváth, Phys. Rev. A55, R3295 (1997).

${ }^{13}$ T. Yamazaki and K.Ohtsuki, Phys. Rev. A45, 7782 (1992).

${ }^{14}$ I. Shimamura, Phys. Rev. A46, 3776 (1992).

${ }^{15}$ N. Morita, K. Ohtsuki and T. Yamazaki, Nucl. Instrum. Methods Phys. Res. Sect. A 330, 439 (1993).

${ }^{16}$ V.I. Korobov and I. Shimamura, Phys. Rev. A56, 4587 (1997).

${ }^{17}$ V.I. Korobov, Phys. Rev. A54, R1749 (1996).

${ }^{18}$ V.I. Korobov, Nucl. Phys. B (Proc. Suppl.) 56 A, 89 (1997).

${ }^{19}$ M. Hori, H.A. Torii, R.S. Hayano, T. Ishikawa, F.E. Mass, H. Tamura, B. Ketzer, F.J. Hartmann, R. Pohl, C. Maierl, T. von Egidy, M. Kumakura, N. Morita, I. Sugai, D. Horváth, E. Widmann, J. Eades and T. Yamazaki, (to be published).

${ }^{20}$ T. Yamazaki, B. Ketzer, E. Widmann, J. Eades, H. Daniel, F.J. Hartmann, M. Hasinoff, R. Pohl, R. Schmidt, T. von Egidy, D. Horváth, M. Kumakura, N. Morita,
I. Sugai, Y. Fujita, H.A. Torii, M. Hori, T. Ishikawa, F.E. Mass, H. Tamura and R.S. Hayano, Chem. Phys. Lett. 265, 137 (1997).

${ }^{21}$ B. Ketzer, F. J. Hartmann, T. von Egidy, C. Maierl, R. Pohl, J. Eades, E. Widmann, T. Yamazaki, M. Kumakura, N. Morita, R. S. Hayano, M. Hori, T. Ishikawa, H. A. Torii, I. Sugai and D. Horváth, Nucl. Phys. B (Proc. Suppl.) 56A, 78 (1997).

${ }^{22}$ B. Ketzer, F.J. Hartmann, T. von Egidy, C. Maierl, R. Pohl, J. Eades, E. Widmann, T. Yamazaki, M. Kumakura, N. Morita, R.S. Hayano, M. Hori, T. Ishikawa, H.A. Torii, I. Sugai and D. Horváth, Phys. Rev. Lett. 78, 1671 (1997).

${ }^{23}$ A. Niestroj, F.J. Hartmann, H. Daniel, B. Ketzer, T. von Egidy, F.E. Maas, R.S. Hayano, T. Ishikawa, H. Tamura, H.A. Torii, N. Morita, T. Yamazaki, I. Sugai, K. Nakayoshi, D. Horváth, J. Eades and E. Widmann, Nucl. Instrum. Methods Phys. Res. Sect. A 373, 411 (1996).

${ }^{24}$ J.E. Kilpatrick, W.E. Keller, E.F. Hammel and N. Metropolis, Phys. Rev. 94, 1103 (1954).

${ }^{25}$ Y. Kawashima, Y. Watanabe and T. Yamazaki, Nucl. Instrum. Methods Phys. Res. Sect. A 274, 241 (1989).

${ }^{26}$ F.J. Hartmann (private communication).

${ }^{27}$ E.U. Condon and G. Breit, Phys. Rev. 49, 229 (1936).

${ }^{28}$ F.J. Hartmann, B. Ketzer, C. Maierl, R. Pohl, T. von Egidy, R.S. Hayano, M. Hori, T. Ishikawa, H. Tamura, H.A. Torii, M. Kumakura, N. Morita, I. Sugai, D. Horváth, J. Eades, E. Widmann and T. Yamazaki, (to be published)

${ }^{29}$ J.B. Hasted, Physics of Atomic Collisions, 2nd edition (Butterworth \& Co., London, 1972), p.21.

30 J. Emsley, The Elements, 3rd edition (Oxford University Press, Oxford, 1996).

${ }^{31}$ P.C. Weast (editor), Handbook of Chemistry and Physics, 70th edition (CRC Press, Boca Raton, 1990).

${ }^{32}$ R. Pohl, F.J. Hartmann, B. Ketzer, T. von Egidy, C. Maierl, J. Eades, E. Widmann, T. Yamazaki, M. Kumakura, N. Morita, R. S. Hayano, M. Hori, T. Ishikawa, H. A. Torii, I. Sugai and D. Horváth, (to be published).

${ }^{33}$ V.I. Korobov (private communication). 
TABLE I. Decay rates $\lambda(n, l)$ of six HAIR daughter states, determined by simultaneously fitting Eq. (3) to a set of analog DATS measured at a temperature $T$ and a pressure $p$ with an $\mathrm{H}_{2}$ admixture $\mathrm{c}_{2}$ as given in the table. The number density of $\mathrm{H}_{2}$ molecules $n_{\mathrm{H}_{2}}$ was calculated using the second order virial expansion of the state equation. The theoretical decay rates $\lambda_{0}(n, l)$ for an isolated $\overline{\mathrm{HHe}}^{+}$system $^{14,16}$, which were used to deduce the quenching cross sections presented in Table II are shown in the last column.

\begin{tabular}{|c|c|c|c|c|c|c|}
\hline$\overline{(n, l)}$ & $T(\mathrm{~K})$ & $p$ (bar) & $c_{\mathrm{H}_{2}}(\mathrm{ppm})$ & $n_{\mathrm{H}_{2}}\left(10^{16} \mathrm{~cm}^{-3}\right)$ & $\lambda(n, l)\left(\mu \mathrm{s}^{-1}\right)$ & $\lambda_{0}(n, l)\left(\mu \mathrm{s}^{-1}\right)$ \\
\hline \multirow[t]{4}{*}{$\overline{(39,36)}$} & 30.2 & 3.02 & 98.6 & 7.1 & $12.4(16)$ & \\
\hline & 30.2 & 4.80 & 98.6 & 11.2 & $21.2(11)$ & \\
\hline & 30.2 & 3.03 & 291 & 21.1 & $34.9(20)$ & \\
\hline & & & & & & 0.62 \\
\hline \multirow[t]{2}{*}{$(39,37)$} & 30.2 & 4.80 & 98.6 & 11.3 & $10.9(10)$ & \\
\hline & & & & & & 0.62 \\
\hline \multirow[t]{3}{*}{$\overline{(39,38)}$} & 29.8 & 3.28 & 291 & 23.1 & $7.6(21)$ & \\
\hline & 29.9 & 4.75 & 291 & 33.3 & $12.7(10)$ & \\
\hline & & & & & & 0.62 \\
\hline \multirow[t]{2}{*}{$(38,35)$} & 30.0 & 1.55 & 968 & 36.2 & $17.0(27)$ & \\
\hline & & & & & & 0.69 \\
\hline \multirow[t]{3}{*}{$(38,36)$} & 30.3 & 1.56 & 968 & 36.1 & $9.1(34)$ & \\
\hline & 30.9 & 4.29 & 291 & 96.8 & $17.3(14)$ & \\
\hline & & & & & & 0.69 \\
\hline \multirow[t]{3}{*}{$(38,37)$} & 33.1 & 8.78 & 968 & 183.3 & $12.1(13)$ & \\
\hline & 31.8 & 9.61 & 968 & 208.8 & $13.8(25)$ & \\
\hline & 29.8 & 10.0 & 968 & 231.3 & $14.2(20)$ & \\
\hline
\end{tabular}

TABLE II. Quenching cross sections $\sigma_{q}(n, l)$ in units of $10^{-16} \mathrm{~cm}^{2}$, obtained from a fit of Eq. (5) to the experimental and theoretical values for the decay rates of Table $\mathrm{I}$.

\begin{tabular}{cc|ccccc}
\hline \hline & $l$ & 34 & 35 & 36 & 37 & 38 \\
$\mathrm{n}$ & & & & & \\
\hline 39 & & $28(10)^{a}$ & $25.8(10)$ & $13.6(13)$ & $5.4(4)$ \\
38 & & $6.8(11)$ & $2.57(21)$ & $0.89(8)$ & \\
37 & $1.1(4)^{a}$ & & & & \\
\hline \hline
\end{tabular}

${ }^{a}$ from Refs. ${ }^{20,21}$ 


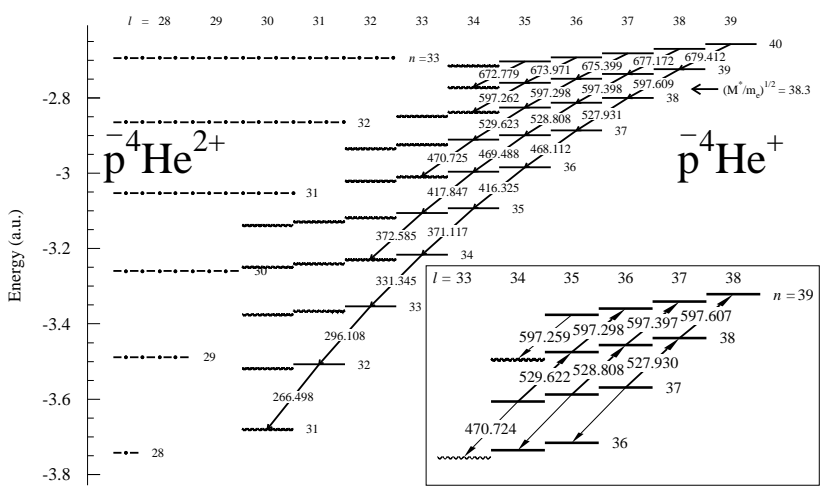

FIG. 1. Level scheme of large- $(n, l)$ states of $\overline{\mathrm{p}}^{4} \mathrm{He}^{+}$. The solid horizontal lines indicate radiation-dominated metastable levels with lifetimes of $\sim 1$ ns, zigzag lines Auger-dominated short-lived states, which decay with a lifetime of $\sim 10 \mathrm{~ns}$ to states of the ionized $\overline{\mathrm{p}}^{4} \mathrm{He}^{2+}$ system $^{14,15}$, shown as dash-dotted lines. Radiative transitions along the main stream of decays with $\Delta v=0$ are displayed as arrows with the theoretical transition wavelengths given in units of $\mathrm{nm}^{17,18}$ The small inset shows an enlarged view of the part of the $(n, l)$ plane, which is covered by our laser spectroscopy experiments, summarizing the six HAIR transitions between normally metastable states (bold arrows) and the two "conventional" transitions from a metastable to a short-lived state at the end of the $v=2$ and $v=3$ cascades (thin arrows). The experimental vacuum wavelengths for these transitions are given in units of $\mathrm{nm}$.

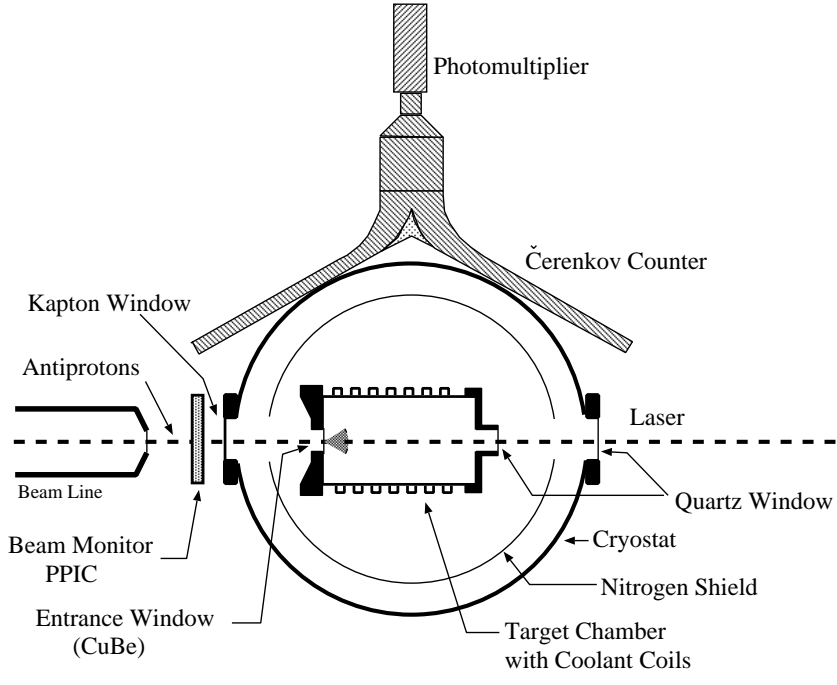

FIG. 2. Plan view of the experimental setup used for the HAIR measurements. The $100 \mathrm{~ns}$ long bunch of antiprotons, extracted from LEAR with a momentum of $200 \mathrm{MeV} / \mathrm{c}$, passed through a position monitor (parallel plate ionization chamber, PPIC) before entering the target chamber through a Kapton and a CuBe window. The target gas with pressures between 1.5 bar and 10 bar was kept at a temperature of $30 \mathrm{~K}$ in a liquid helium cryostat. The charged annihilation products were detected by a Čerenkov counter on one side of the target vessel, the PMT being read out by a digital oscilloscope. The pulsed laser beam entered the target through quartz windows on the opposite side of the chamber. 


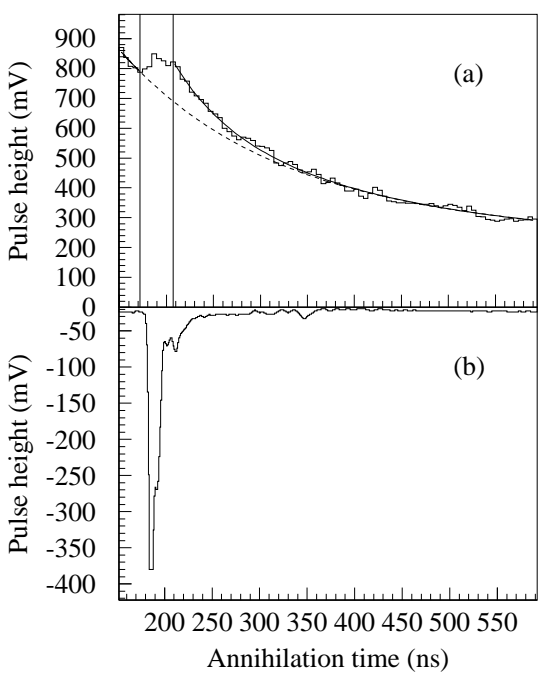

FIG. 3. (a) Analog DATS (photomultiplier output pulse shape as recorded by a digital oscilloscope) of the transition $(n, l)=(38,35) \rightarrow(39,36)$ obtained at $30.2 \mathrm{~K}, 4.8 \mathrm{bar}$ and an $\mathrm{H}_{2}$ concentration of $98.6 \mathrm{ppm}$. The solid line is the result of simultaneously fitting Eq. (3) to a set of 27 analog DATS (including the one displayed), the dashed line represents the "background" function Eq. (2) without the laser-induced peak. The vertical lines indicate the duration of the laser pulse, which has been excluded from the fit. (b) Time structure of the corresponding laser pulse recorded by a PIN diode.

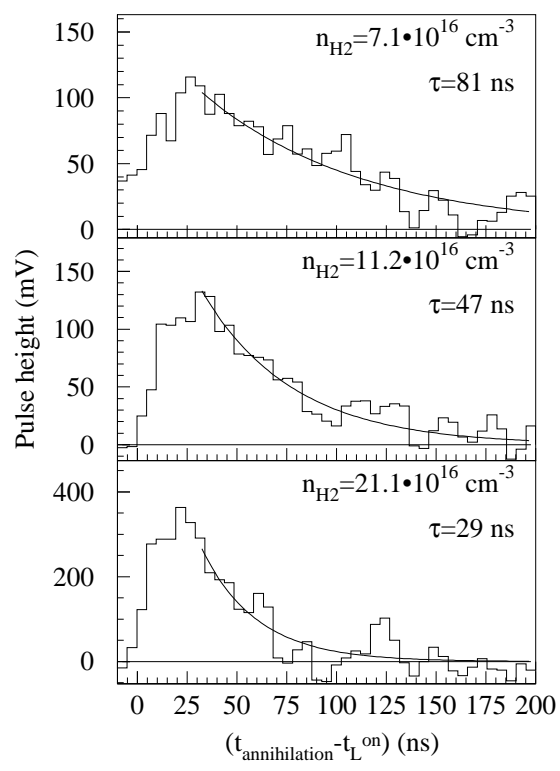

FIG. 4. Background subtracted HAIR peaks of the transition $(n, l)=(38,35) \rightarrow(39,36)$ at three different number densities $n_{\mathrm{H}_{2}}$ of $\mathrm{H}_{2}$ molecules. The lifetime $\tau$ of the peak, directly reflecting the lifetime of the daughter state of the transition, decreases with increasing $\mathrm{H}_{2}$ admixture.

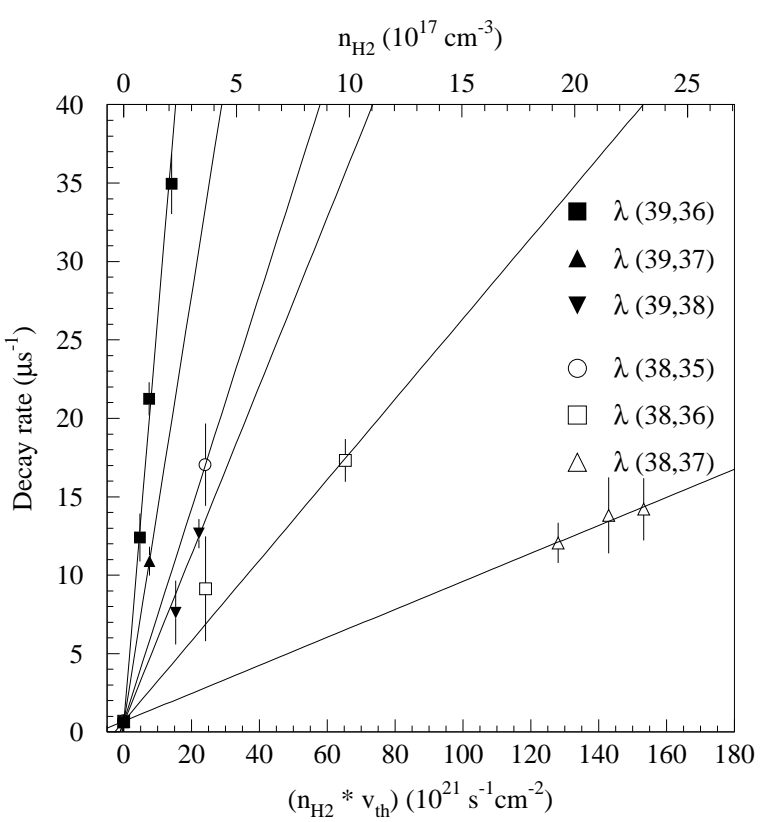

FIG. 5. Decay rates of the six HAIR transitions for different $\mathrm{H}_{2}$ number densities. To account for small fluctuations of the temperature between the different measurements, the decay rates are plotted versus the flux $n_{\mathrm{H}_{2}}(T) v_{\mathrm{th}}(T)$. The corresponding approximate number density of $\mathrm{H}_{2}$ molecules is indicated on the top abscissa. The straight lines show the results of fitting Eq. (5) to the theoretical and experimental decay rates of Table $\mathrm{I}$. 


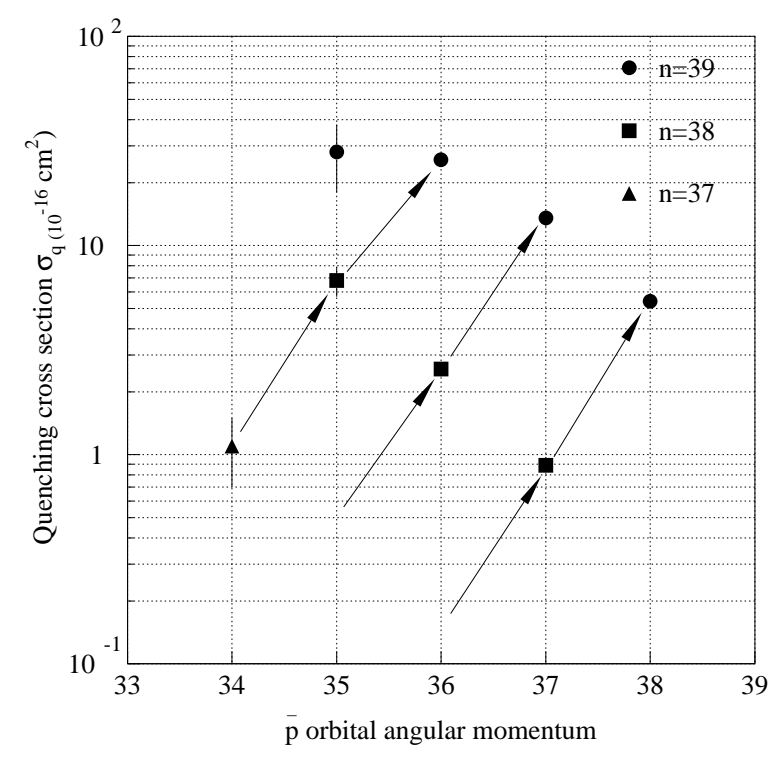

FIG. 6. The $(n, l)$ dependence of the $\mathrm{H}_{2}$ quenching cross section $\sigma_{\mathrm{q}}$. The arrows indicate HAIR transitions between a long-lived lower state and an $\mathrm{H}_{2}$-induced short-lived upper state.

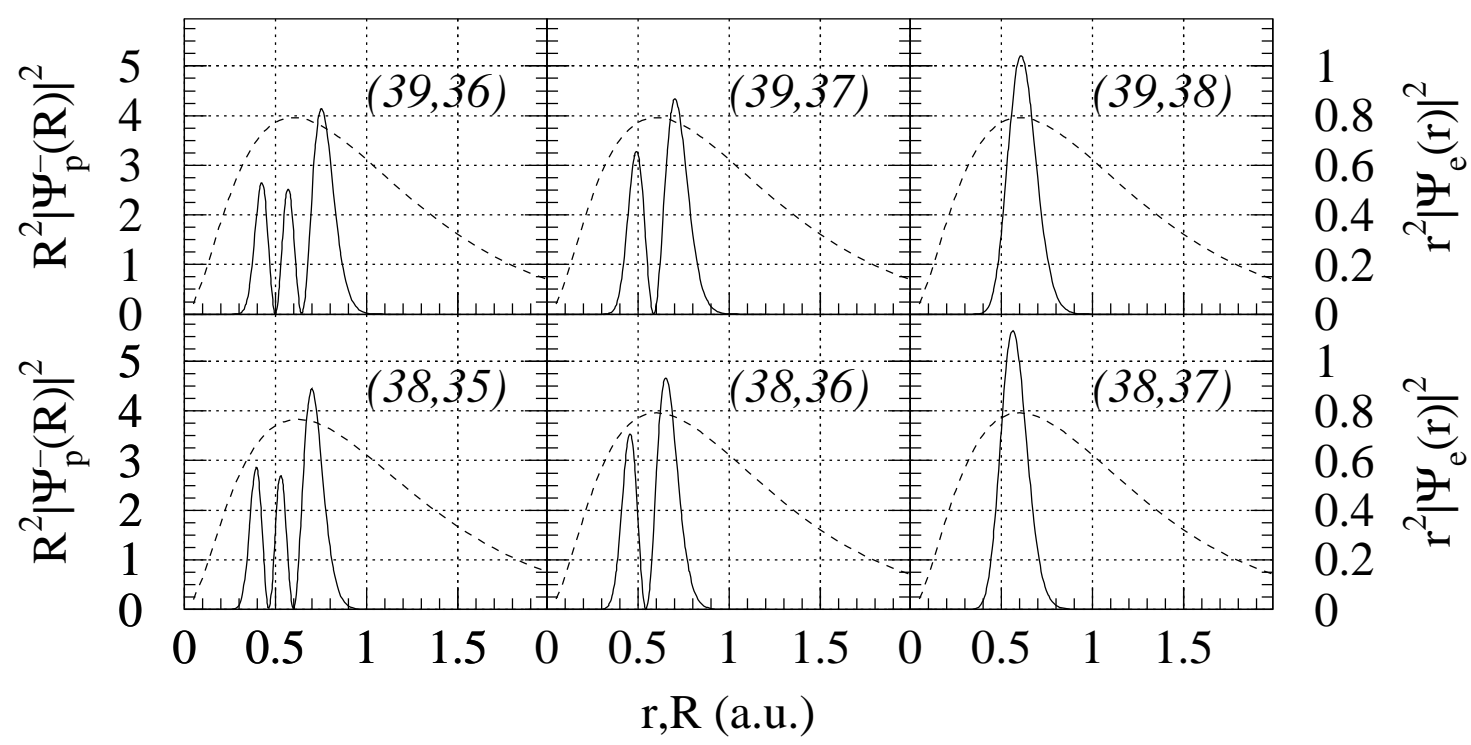

FIG. 7. Radial probability densities of the antiproton $R^{2}\left|\Psi_{\overline{\mathrm{p}}}(R)\right|^{2}$ (solid curve) and the electron $r^{2}\left|\Psi_{e}(r)\right|^{2}$ (dashed curve) for each of the six HAIR daughter states, calculated by Korobov ${ }^{17,18,33}$, as a function of the $\overline{\mathrm{p}}-\mathrm{He}^{2+}$ distance $R$ and the $\mathrm{e}^{-}-\mathrm{He}^{2+}$ distance $r$. All angular variables have been integrated over, thus neglecting the core polarization effect of the electron cloud due to the repulsive interaction between the antiproton and the electron ${ }^{13}$. 